\title{
Le radar météorologique de Trappes et l'estimation des intensités pluvieuses en Seine-Saint-Denis. Intérêt pour la gestion des réseaux d'assainissement.
}

\author{
Trappes' weather radar and rainfall intensities in Seine-Saint-Denis
}

H. Andrieu

Laboratoire central

des Ponts et Chaussées
G. Jacquet

CERGRENE

ENPC

Le projet de gestion automatisée du réseau d'assainissement de Seine-St-Denis nécessite l'amélioration des techniques de mesure et de prévision des intensités pluvieuses. Les réseaux de pluviographe présentent en effet des insuffisances que le radar météorologique est susceptible de combler.

L'étude que relate cet article, cherche à évaluer la capacité du radar à déterminer les précipitations pour les besoins de l'hydrologie urbaine; elle s'appuie sur une campagne de mesures de deux mois au cours de l'été 1982. Après une analyse qualitative des données recueillies, diverses techniques d'ajustement des images radar sont testées. Les résultats obtenus sont comparés à ceux déduits d'une interpolation des mesures pluviographiques seules. Le role du radar dans le projet de gestion automatisée est esquissé. Des essais de simulation de débits sur des bassins versants urbains sont effectués après une approche de l'influence du modèle de ruissellement vis-à-vis des incertitudes de mesures des précipitations.

The computer-assisted management scheme concerning the storm sewer system requires that the measurement and rainfall intensity forecast techniques be improved. The recording rain gauge networks have insufficiencies which should be made up for by weather radar.

The study relating to this article seeks to assess the radar capacity for determining precipitations concerning the requirements of urban hydrology and this is based on a two month' 'campaign conducted during the Summer of 1982. Following a qualitative analysis of the data collected, various adjustment techniques relating to radar images were tested. The results obtained were compared with those deduced from an interpolation of rain gauge measurements alone. The role of the radar as part of the computer-assisted management scheme is being delineated. Simulation tests concerning the flow rates in urban catchment basins were carried out after an approximation of the effect of the surface water model in relation to precipitation measurement uncertainities was made.

\section{Introduction}

Le développement des agglomérations urbaines s'accompagne d'une imperméabilisation croissante des sols et se traduit, en cas de pluie, par un accroissement du ruissellement. L'insuffisance des réseaux d'assainissement qui peut en résulter entraîne ainsi l'inondation répétée de zones habitées. La solution la plus courante pour résoudre ce problème consiste à augmenter la capacité des collecteurs : elle est coûteuse et se révèle parfois techniquement difficile. Aussi, les services gestionnaires de grands ré- seaux d'assainissement essaient d'utiliser au mieux les capacités des ouvrages existants : collecteurs, bassins de retenue, déversoirs d'orage...

Dans cette perspective, le département de SeineSaint-Denis met progressivement en place un important projet de gestion automatisée du réseau d'assainissement. La gestion automatisée par temps de pluie, vise à réguler, en temps réel, les débits transitant par les ouvrages de façon à en optimiser les conditions de fonctionnement. La diminution de la fréquence et de l'importance des inondations et la réduction des rejets polluants au milieu naturel sont ainsi les principaux objectifs poursuivis. 
Une bonne connaissance des précipitations et de leur évolution occupe une place prépondérante dans la définition des stratégies de gestion du réseau. En SeineSaint-Denis, cette connaissance est assurée par un réseau de pluviographes télétransmis dont la densité avoisine un appareil pour 15 à $20 \mathrm{~km}^{2}$. Il se révèle qu'un tel réseau est mal adapté à la détection des orages d'été, intenses et souvent très localisés, qui provoquent de nombreuses inondations en milieu urbain. L'accroissement du nombre de pluviographes reste envisageable, mais alourdit notablement les contraintes d'entretien du parc d'appareils. De plus, un réseau de mesure dense, mais ne couvrant que quelques centaines de kilomètres carrés, n'apporte qu'une information fragmentaire sur les précipitations et ne transmet aucune indication sur leur évolution à très courte échéance : quelques heures au plus; or cet aspect est important pour l'efficacité de la gestion du réseau.

Le radar météorologique, instrument de télédétection des précipitations offre de nouvelles perspectives dans le domaine de la mesure et de la prévision des intensités pluvieuses. En effet, les images radar numérisées permettent un suivi continu et immédiatement centralisé des zones pluvieuses. Le but de l'étude entreprise en SeineSaint-Denis est d'évaluer l'intérêt du radar en hydrologie urbaine. S'appuyant sur une quantité de données limitée, elle ne constitue toutefois qu'une première étape qui devra être ultérieurement poursuivie et approfondie.

L'utilisation hydrologique du radar météorologique se développe lentement depuis une vingtaine d'années environ. Les principales applications concernaient initialement l'évaluation de lames d'eau moyennes sur de vastes superficies (Wilson, Brandes - 1979). D'importantes études ont été engagées en Grande-Bretagne dans le domaine de l'annonce et de la prévision des crues (Dee Weather Radar Project-1977, Collier et al.-1980, Collier-1986). Au début des années 80 , l'expérience française, moins étoffée, englobe la surveillance qualitative du bassin versant de la Dordogne (Dupouyet - 1983) et le projet Hydromel de la Météorologie Nationale (Froment-1979). Ce dernier organisme constitue actuellement un réseau national de radars météorologiques: projet Aramis (Gilet et Ciccione - 1983) et rediffuse les images radars. L'intérêt du radar est donc relancé aux yeux des hydrologues. Parallèlement à l'étude ici décrite, une autre expérience s'est déroulée dans le Bassin Parisien, à partir du radar de Dammartin (Delrieu - 1986);

Le rôle du radar à l'échelle d'une agglomération urbaine a notamment été étudié à Montréal (Austin et al. - 1974) et à Chicago (Huff et Towery-1978), mais ne s'est pas concrétisé au stade opérationnel. La particularité de l'hydrologie urbaine est provoquée par les caractéristiques des bassins versants, petits et très imperméabilisés, dont le temps de réponse excède rarement quarante cinq minutes à une heure. Les conditions d'utilisation des données radar sont donc contraignantes : les intensités de la pluie doivent être évaluées sur des pas de temps courts (10 à 15 minutes) à l'échelle quasi-ponctuelle de la maille radar. Pour le gestionnaire d'un grand réseau d'assainissement, l'évaluation des intensités pluvieuses ne constitue qu'une étape, et l'intérêt du radar réside dans l'aide qu'il peut offrir pour appréhender en temps réel ou pour prévoir les débits à l'exutoire d'un bassin versant urbain.

\section{Le radar météorologique et la détection des précipitations}

\subsection{Fonctionnement du radar météorologique}

Une présentation complète du radar météorologique et de ses applications est affectuée par Sauvageot, 1982.

Rappelons que le radar est un instrument de détection et de mesure basé sur l'émission régulière de brèves impulsions électromagnétiques de forte puissance. La rencontre d'un obstacle provoque la diffusion des ondes incidentes dans toutes les directions, une partie : le signal utile, étant rétrodiffusée vers l'antenne du radar. La connaissance de l'orientation de l'antenne, le mesure de l'intervalle de temps entre l'émission et la réception en retour assurent la localisation de la cible qui est, de plus, caractérisée par la puissance du signal réfléchi. Dans le cas des précipitations, cette cible regroupe l'ensemble des gouttes d'eau présentes dans le volume d'atmosphère sondé par le faisceau. L'aptitude de cette cible à diffuser l'énergie incidente s'exprime par un facteur de réflectivité radar, noté $Z$, tel que $Z=\sum D_{i}^{6}$ où $D_{i}$ décrit le diamètre de l'ensemble des gouttes d'eau du volume considéré. La puissance rétrodiffusée $(p$ ) est la grandeur effectivement mesurée, elle est reliée au facteur de réflectivité radar par la relation :

$$
p_{r}=C \cdot L(r) \cdot \frac{Z}{r^{2}}
$$

avec

$r \quad$ : distance du radar à la cible

$C$ : constante caractéristique du radar

$L(r)$ : fonction d'atténuation du signal avec la distance

En première approximation, et pour les longueurs d'onde adaptées à la mesure des précipitations $(5 \mathrm{~cm}$ à $10 \mathrm{~cm}$ ), on admet que $L(r)=1$.

Le traitement du signal reçu par l'antenne permet d'attribuer à un volume élémentaire la quantité d'énergie qu'il rétrodiffuse. L'information définitive prend la forme d'images numérisées de réflectivités exprimées en dBZ.

\subsection{Principe de la mesure des précipitations}

Des relations expérimentales entre l'intensité de la pluie $(\mathrm{R}$ en $\mathrm{mm} / \mathrm{h}$ ) et la réflectivité radar ont été proposées. Elles prennent la forme $Z=A R^{h}$ où $A$ et $b$ sont des paramètres dépendant de l'origine météorologique des précipitations. La loi de Marshall-Palmer est la plus utilisée d'entre elles : $Z=200 R^{1.6}$. Les travaux effectués (Wilson, Brandes - 1979) montrent cependant que l'évaluation des intensités pluvieuses par ces relations reste approximative. En effet un certain nombre d'erreurs peuvent entacher la mesure radar. Parmi celles-ci :

- la mesure des réflectivités peut être faussée (effet de masques, propagation anormale du faisceau, atténuation de ce dernier...)

- la relation $Z=A R^{b}$ choisie a priori n'est pas adaptée - l'altitude du faisceau s'accroît avec l'éloignement du radar et la représentativité de la mesure de réflectivité se dégrade...

L'utilisation conjointe des images radar et des mesures pluviographiques est alors envisagée pour remédier à 


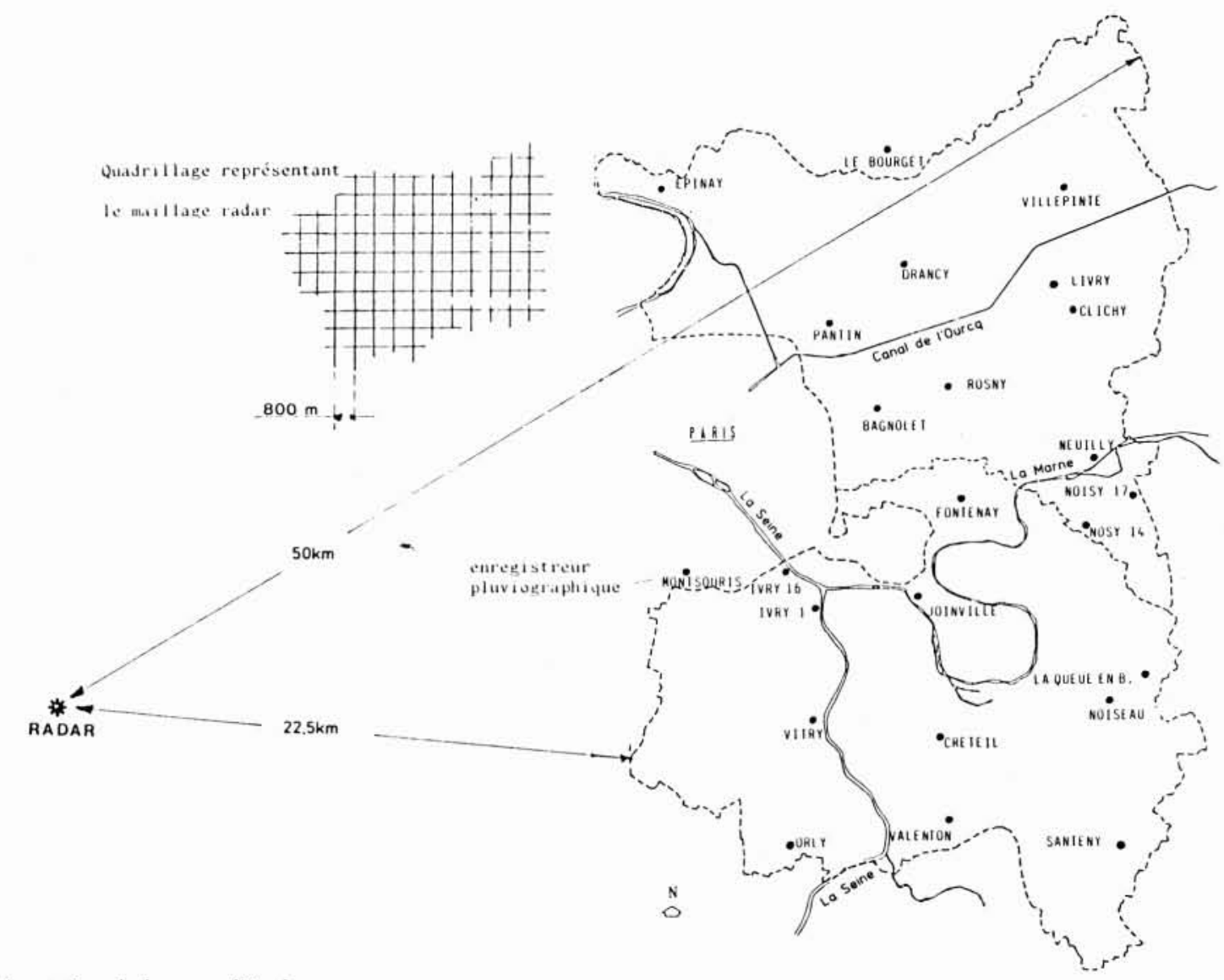

1. Présentation de la zone d'étude.

certaines causes d'erreur. Séduisante, elle conjugue la qualité ponctuelle de la mesure pluviographique et la continuité spatiale de l'image radar. Cette démarche : le calibrage ou l'ajustement des images radar par des pluviographes permet effectivement d'améliorer la qualité de l'estimation radar (Harrold et al. - 1974). Plusieurs méthodes de calibrage ont été proposées.

L'image radar est tout d'abord exprimée en intensité de la pluie au moyen d'une loi : $Z=A R^{\prime \prime}$ a priori adaptée. Aux points d'implantation d'un pluviographe sont alors disponibles l'intensité pluviographique (IP) et l'intensité radar sur la maille correspondante $(I R)$.

Dans l'hypothèse la plus simple, où la correction appliquée à l'image est considérée comme uniforme, un facteur de corrrection moyen est évalué :

$$
f c_{i}=\sum_{i} I P_{i} / \sum_{i} I R_{i} \quad \text { ou } \quad f c=\frac{1}{n} \sum I P_{i} / I R_{i}
$$

Il est également possible de prendre en compte les variations du facteur de correction dans l'espace. Dans cette hypothèse, calibrer l'image radar consiste à appliquer un schéma d'interpolation aux facteurs de correction déterminés aux divers points de comparaison.

Dans tous les cas, l'intensité résultante de la pluie en un point $M_{0}$ s'exprime : $I_{0}=f c_{0} \times I R_{0} ; f c_{0}$ étant le facteur correctif pour la maille radar $M_{0}$.

\section{Expérience en Seine-Saint-Denis durant l'été 1982}

Les Services de la Météorologie nationale ont, au cours de l'été 1982, assuré la couverture radar d'une zone d'étude, dont la superficie avoisine $500 \mathrm{~km}^{2}$, qui regroupe les deux départements de Seine-Saint-Denis et du Val-de-Marne ( figure I). Le radar Rodin, de Trappes, d'une longueur d'onde de $5 \mathrm{~cm}$, a été utilisé à cet effet (David - 1980).

Cette surface est instrumentée par 22 enregistreurs pluviographiques et des limnigraphes surveillent l'évolution des débits à l'exutoire de plusieurs bassins versants. L'expérience a bénéficié de conditions très favorables à l'évolution de l'intérêt du radar en hydrologie urbaine :

- la proximité entre le radar et la zone d'étude $(22 \mathrm{~km}$ à $50 \mathrm{~km}$ ) permet de limiter les risques d'erreur dans la mesure radar,

- les images radar sont acquises à la fréquence d'une toutes les deux minutes sur seize niveaux de réflectivités. Chaque maille représente un carré de $800 \mathrm{~m}$ de côté. Cette résolution spatiale est proche de la superficie des petits bassins versants urbains. Il apparaît toutefois que la numérisation sur seize niveaux est insuffisante pour les applications hydrologiques,

- les pluviographes, non télétransmis à cette date, ont été 

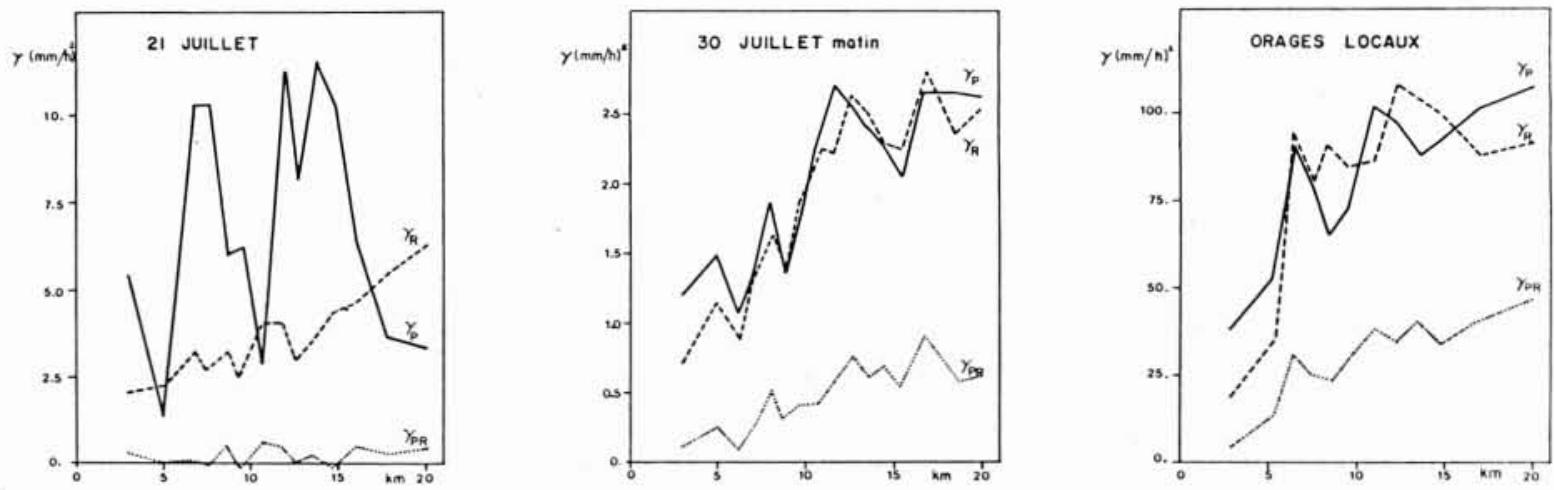

2. Variogrammes radar, pluviographique et croisé.

soigneusement suivis pour assurer une bonne synchronisation avec le radar; un pas de temps de travail de 15 minutes peut être envisagé.

Il a été ainsi possible d'enregistrer dans de bonnes conditions le déroulement de quatre séquences d'origines météorologiques diversifiées: pluie régulière de front chaud, orages convectifs très localisés, averse très étendue à caractère convectif.

\section{Radar météorologique et estimation des inten- sités pluvieuses}

L'évaluation de l'intérêt du radar pour déterminer les intensités pluvieuses, dans des conditions adaptées à l'hydrologie urbaine, est guidée par deux lignes directrices :

- les tâches qui participent au projet de gestion s'effectuent en temps réel. Il en est de même de l'acquisition et du traitement des images radar. La confirmation du rôle du radar et les perspectives opérationnelles conduiront donc dans une première étape, à adopter une méthode d'ajustement des images radar simple et robuste,

- cette perspective opérationnelle n'exclut pas une analyse plus complète des données recueillies. Dans cette optique, il est nécessaire de mettre en œuvre une technique d'ajustement qui exploite au mieux l'information disponible. Cette méthode sera considérée comme une sorte de référence à laquelle seront comparées les méthodes plus courantes d'ajustement des images radar, susceptibles d'un usage en temps réel. Le krigeage, technique d'interpolation basée sur l'étude préalable de la structure spatiale du champ traité, a été adopté à cet effet (Matheron - 1970, Delhomme - 1976).

\subsection{Cohérence des mesures radar et pluviographiques}

L'ajustement des images radar repose sur l'utilisation conjointe des données radar et pluviographiques. Cette démarche n'a cependant de sens que si ces deux capteurs décrivent de façon cohérente le champ des intensités pluvieuses; cet aspect doit donc être vérifié.

a) La simple comparaison visuelle des hyétogrammes pluviographiques et, au même point, de la mesure radar, apporte quelques indications. Malgré la surestimation significative entraînée par l'utilisation, a priori, de la loi $Z=200 R^{16}$, la prise en compte de l'allure des courbes met en évidence leur bonne concordance : les heures de début et de fin de pluie, la position des pics d'intensité coïncident assez généralement. Des anomalies sont cependant constatées, à l'occasion des orages très localisés. En particulier sur le pourtour des cellules pluvieuses, des échos radar significatifs peuvent être observés en l'absence de précipitations au sol (malgré la faible altitude de l'axe du faisceau).

b) La cohérence entre les deux types de mesure peut également être appréhendée en s'intéressant à la structure spatiale des précipitations.

La variabilité d'un champ $z$ qui se déploie dans l'espace est décrite par son variogramme (noté $\gamma_{\text {: }}$ ):

$$
\gamma_{:}(h)=\frac{1}{2} \operatorname{Var}[z(u+h)-z(u)]
$$

Cette courbe traduit la façon dont l'information contenue dans une mesure ponctuelle se dégrade au fur et à mesure que l'on s'en éloigne. Dans le cas des intensités pluvieuses, et du fait de la présence de deux techniques de mesure, considérons les deux champs, pluviographique et radar. Nous avons alors :

- un variogramme pluviographique $\left(\gamma_{P}\right)$ déduit des données pluviographiques

- un variogramme radar $\left(\gamma_{R}\right)$ correspondant, rendu homogène au précédent par l'adoption d'un facteur correctif de l'image qui efface la surestimation systématique.

La comparaison de ces deux variogrammes permet de distinguer d'éventuelles divergences dans la façon dont les deux techniques de mesures perçoivent le champ pluvieux (figure 2). C'est notamment le cas lors de la pluie du 21 juillet, pour laquelle la courbe radar met en évidence une structure qui n'apparait pas sur la courbe pluviographique. Dans les deux autres cas, 30 juillet et orages locaux, les 


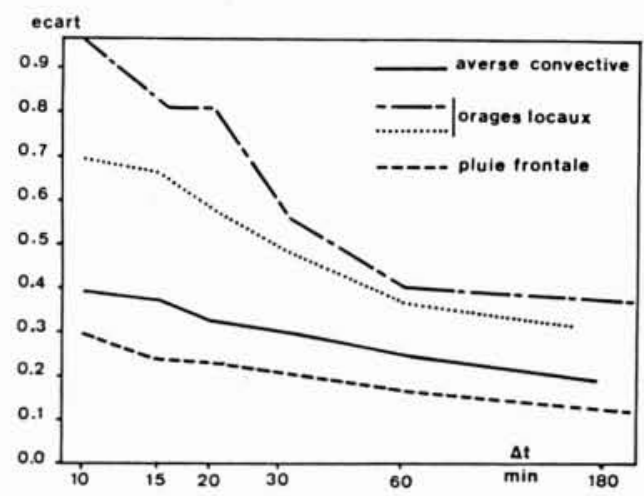

3. Evolution des écarts selon le pas de temps.
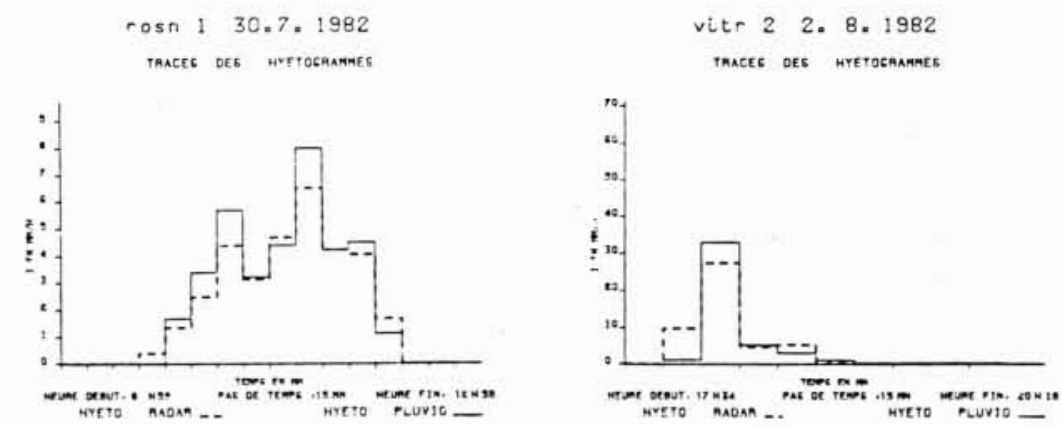

4. Exemples de hyétogrammes reconstitués à l'aide du radar. deux variogrammes expérimentaux sont voisins. Cette bonne concordance ne permet cependant pas de conclure à la cohérence des deux techniques de mesures. Elle doit être complétée par l'étude de la cofluctuation des deux champs radar et pluviographique. Leur variogramme croisé, noté $\gamma_{R A}$ est utilisé à cet effet :

$\gamma_{R P}(h)=\frac{1}{2} E[(I R(u+h)-I R(u)) \cdot(I P(u+h)-I P(u))]$

oủ $I R$ et $I P$ représentent respectivement les intensités radar et pluviographiques. Le variogramme croisé s'interprète comme une fonction de covariance. Pour l'exemple qui nous intéresse, où $\gamma_{R}$ et $\gamma_{P}$ sont voisins, la cohérence entre les champs sera d'autant plus nette que $\gamma_{R}$ r sera proche de ces deux courbes. La figure 2 confirme que pour la pluie du 21 juillet les deux sources de données sont assez peu cohérentes. Lors du 30 juillet matin et des orages locaux la cofluctuation entre les mesures radar et pluviographiques est plus significative, même si seulement $30 \%$ de la variance environ de l'un des champs est expliquée par l'autre dans le premier cas et $40 \%$ lors des orages.

c) Calibrer des images radar impose d'assimiler aux points de comparaison, la donnée pluviographique ponctuelle à l'intensité radar sur une maille qui recouvre 64 hectares. Une incertitude est ainsi introduite, difficile à ipréciser dans la mesure où nous connaissons très mal la structure des précipitations à très petite échelle. Cette incertitude s'accroit avec la variabilité des précipitations : elle est donc plus importante pour les pas de temps courts et à l'occasion des orages très localisés. Des essais ont montré dans ce dernier cas que, pour un pas de temps de 15 minutes, l'écart moyen entre la mesure ponctuelle et la valeur moyenne sur une maille peut atteindre $30 \%$.

\subsection{Résultats obtenus par calibrage des images radar}

\section{a) Présentation}

A la différence de l'approche classique esquissée au paragraphe 2.2 , on ne raisonnera pas en termes de facteurs de correction, mais directement sur la quantité $\log A=$ $\log Z-b \cdot \log I P$, qui peut être évaluée en chaque point où coexistent les données radar et pluviographiques. Le paramètre $b$ restera quant à lui inchangé $(b=1.6)$; on a en effet vérifié que ses variations influent peu sur la qualité du résultat définitif. Le choix de $\log A$ a été effectué de façon à travailler sur un paramètre normalement distribué, dont les fluctuations par rapport à la valeur moyenne restent réduites, ce qui n'est pas le cas du facteur de correction.

L'interpolation des valeurs du paramètre $\log A$ a été effectuée en utilisant le krigeage. Cette technique, maintenant bien connue, ne sera pas détaillée ici. Après une analyse de la structure spatiale du champ étudié, l'estimation en chaque point est recherchée sous la forme d'une combinaison linéaire des différentes mesures ponctuelles, sans biais et de variance minimale (Matheron, Delhomme).

Si $\log A_{0}$ est la valeur estimée du paramètre de calibrage sur la maille $M_{0}$, et $Z_{0}$ la réflectivité mesurée en en point, l'intensité de la pluie sera donc $\tilde{I}_{0}=\left(Z_{0} / \tilde{A}_{0}\right)^{1 / \hbar}$. L'image radar est recalibrée à chaque pas de temps. Seules les mailles radar oủ est présente une donnée pluviographique peuvent être prises en compte pour apprécier la pertinence de l'intensité pluvieuse évaluée à partir du radar. Une procédure de validation croisée a été adoptée à cet effet : les intensités de la pluie sur une maille recouvrant un pluviographe sont reconstituées au moyen du radar calibré par les autres pluviographes. Cette procédure est répétée à tous les points de mesure au sol. L'indicateur suivant a été retenu pour évaluer la qualité de l'estimation :

$$
\text { avec } \quad E=\frac{\Sigma\left[I P_{i j}-I R_{i j}\right]}{\Sigma I P_{i j}}
$$

IP : Mesure pluviographique

IR : estimation radar

$i$ et $j$ : indices décrivant l'ensemble des pas de temps et des points de mesures au sol.

Compte tenu des remarques précédentes, il advient que la quantité $E$ ne représente pas exactement l'erreur liée à l'estimation radar.

\section{b) Résultats (fig. 3 et 4 )}

La figure 3 regroupe les résultats obtenus pour chaque 

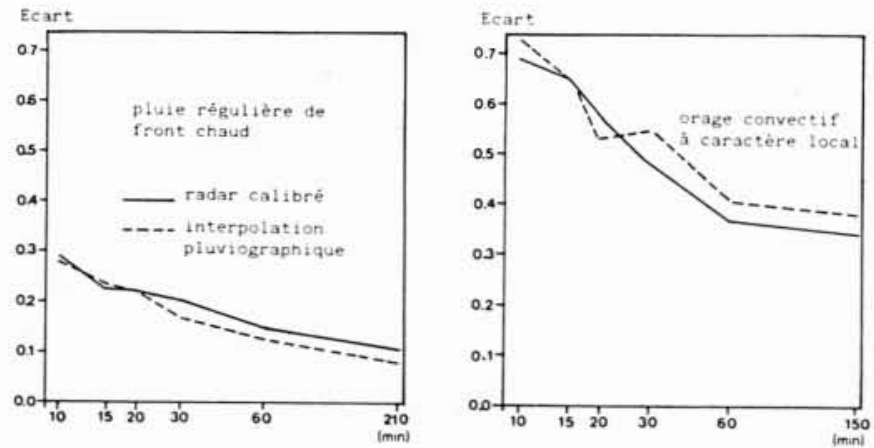

5. Comparaison entre radar calibré et interpolation pluviographique.
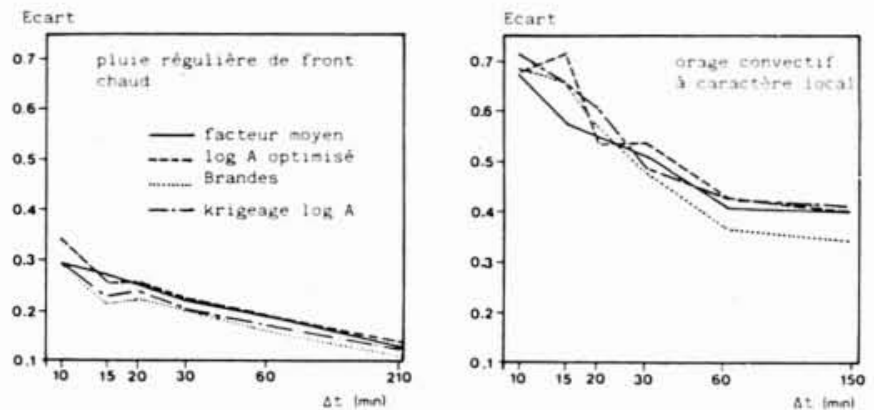

6. Comparaison des méthodes d'ajustement des images radar
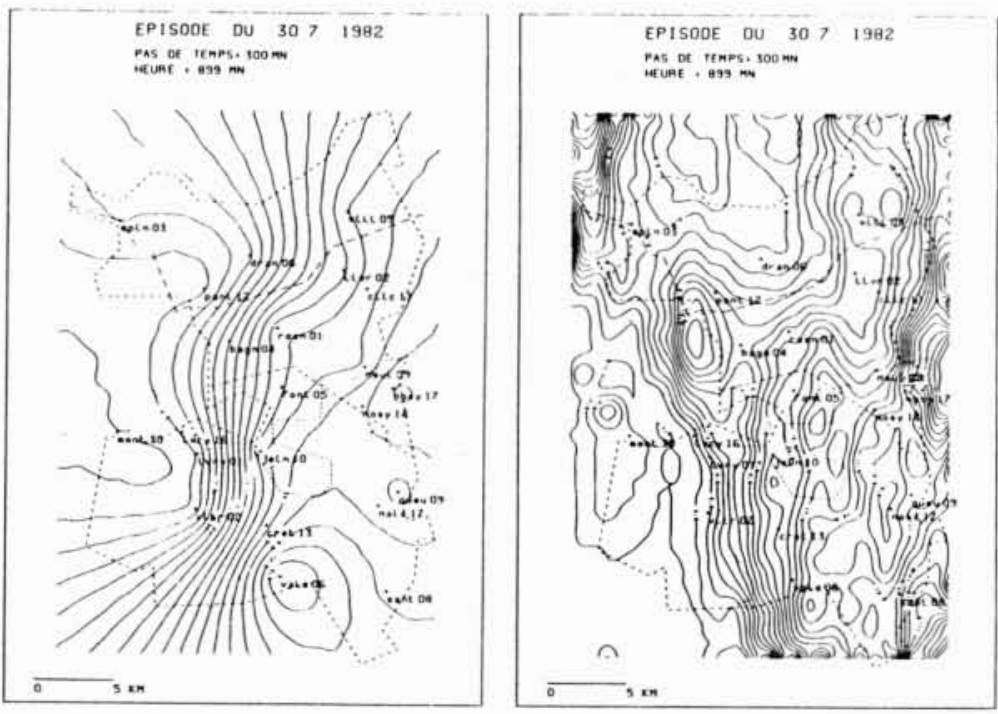

7. Cartographie des lames d'eau lors de l'orage du 30 juillet au soir. 
séquence pluvieuse et présente l'évolution de l'indicateur d'écart en fonction du pas de temps d'exploitation des données. A titre illustratif la figure 4 compare des hyétogrammes pluviographique et radar.

Il apparait ainsi que les écarts de reconstitution sont directement liés à la variabilité spatiale des intensités pluvieuses et à leur origine météorologique. Les orages convectifs à caractère local sont en effet plus difficiles à décrire que les pluies régulières. Cette constatation montre que la continuité spatiale introduite par le radar dans la perception des précipitations n'est que partiellement exploitable en termes d'intensité de la pluie.

\subsection{Comparaison entre calibrage des images radar et interpolation pluviographique}

En l'absence de radar météorologique, seule l'interpolation des mesures pluviographiques permet, à l'intérieur d'un réseau d'appareils, d'approcher l'intensité de la pluie en un point. En appliquant la méthode d'interpolation : le krigeage, à ces mesures pluviographiques seules, nous déduisons les résultats que l'on obtient sans le radar. La même procédure de validation croisée et le même critère d'écart ont naturellement été utilisés pour comparer, aux points internes du réseau, le résultat de l'interpolation pluviographique sur la maille radar à la mesure effectuée en ce point.

La figure 5 illustre la comparaison de ces deux approches, images radar calibrées d'une part, réseau de pluviographes d'autre part. Les courbes obtenues montrent que pour les quatre pluies étudiées en Seine-SaintDenis et en s'attachant à des évaluations quasi-ponctuelles à l'intérieur du réseau de mesure existant, calibrage des images radar et interpolation de données pluviographiques conduisent à des résultats voisins.

\subsection{Comparaison des méthodes de calibrage}

En conservant la démarche décrite précédemment, les méthodes courantes de calibrage des images radar ont été testées :

- facteur moyen de correction constant sur l'image: après une première estimation des intensités radar déduite de la loi $Z=200 R^{1.6}$, le facteur de correction $f c=$ $\Sigma I P / \Sigma I R$ permet de corriger ces valeurs.

- recherche de la valeur du paramètre $A$, constante sur toute l'image qui minimise la forme quadratique

$$
Q=\sum_{i=1}^{i n} I P_{i} \cdot\left[I P_{i}-I R_{i}\right]^{2}
$$

avec $I R_{i}=\left(\mathrm{Z}_{i} / A\right)^{1 / \hbar}$ et $i$ décrivant les points de comparaisons radar-pluviographes.

- technique de calibrage proposée par Brandes (1975) qui connait un certain succès. En tout point $M_{0}$ de l'image, une estimation du paramètre de calibrage $\tilde{p}_{0}$ est donnée par :

$$
\tilde{p}_{0}=\left(\sum_{k=1}^{n} W_{o k} \cdot p_{k}\right) /\left(\sum_{k=1}^{n} W_{o k}\right) \text { et } W_{o k}=\exp \left(-\frac{d_{o k}^{2}}{D^{2}}\right)
$$

$p_{k}$ : paramètre de calibrage au point de référence $k$

$d_{0 k}$ : distance entre $M_{0}$ et le point $k$

$D$ : paramètre de réglage.
En chaque point de référence la méthode génère $\tilde{p}_{k}$ différent de $p_{k}$. La procédure est appliquée aux écarts $\left(p_{k}\right.$ $\tilde{p} k$ ) avec $D^{\prime}=\mathrm{D} / 2$. En définitive

$$
p_{0}=\tilde{p}_{0}+\left[\sum_{k=1}^{k=1} W_{n k}^{\prime} \cdot\left(p_{k}-\tilde{p}_{k}\right)\right] / \sum_{k=1}^{k=1} W^{\prime}{ }_{n k}
$$

La comparaison de ces méthodes est illustrée par la figure 6, qui reprend également le calibrage des images radar par krigeage de $\log A$. Il se révèle, dans les conditions propres à la Seine-Saint-Denis et pour les pluies étudiées, que les méthodes testées conduisent à des résultats assez proches. La prise en compte de la variabilité semble bénéfique pour les pluies régulières, bien structurées. A l'occasion des orages locaux et notamment lors des pas de temps courts $(\Delta t=15 \mathrm{mn})$, cet avantage disparait.

\subsection{Cartographie des lames d'eau}

De façon à appréhender l'intérêt du radar, l'accent a été mis sur l'évaluation d'intensités au niveau de la maille. Or, l'information radar qui prend la forme d'une image, contient une information beaucoup plus riche. Elle permet notamment, à l'échelle de la zone d'étude, de visualiser et de représenter les intensités pluvieuses. Il est ainsi possible de mettre en parallèle la cartographie des lames d'eau :

- déduite des images radar ajustées par un facteur moyen de correction

- obtenue en interpolant les données du réseau pluviographique.

Cette comparaison reste cependant qualitative dans la mesure où aucune référence ne peut être proposée. Elle est illustrée par la figure 7 , consacrée à la cartographie des lames d'eau précipitées au cours de l'orage du 30 juillet au soir. L'allure générale des isohyètes est assez voisine sur les deux cartes qui diffèrent néanmoins sur deux aspects :

- l'aspect plus détaillé de la carte radar, dont il n'est cependant pas possible de vérifier la pertinence,

- la forme des courbes isohyètes à l'extérieur du réseau de pluviographes. Cette constatation traduit la perception beaucoup plus générale qu'offre le radar du champ pluvieux. Elle introduit le rôle que peut jouer cet instrument comme outil de prévention et de prévision des précipitations.

\section{Participation du radar météorologique à la gestion d'un réseau d'assainissement}

Une description détaillée du projet de gestion automatisée de Seine-Saint-Denis est effectuée par Bachoc, 1984. Dans un tel projet, le radar est susceptible de présenter un double intérêt, tout d'abord en tant qu'instrument de prévention, ensuite comme instrument de mesure et de prévision à courte échéance. 

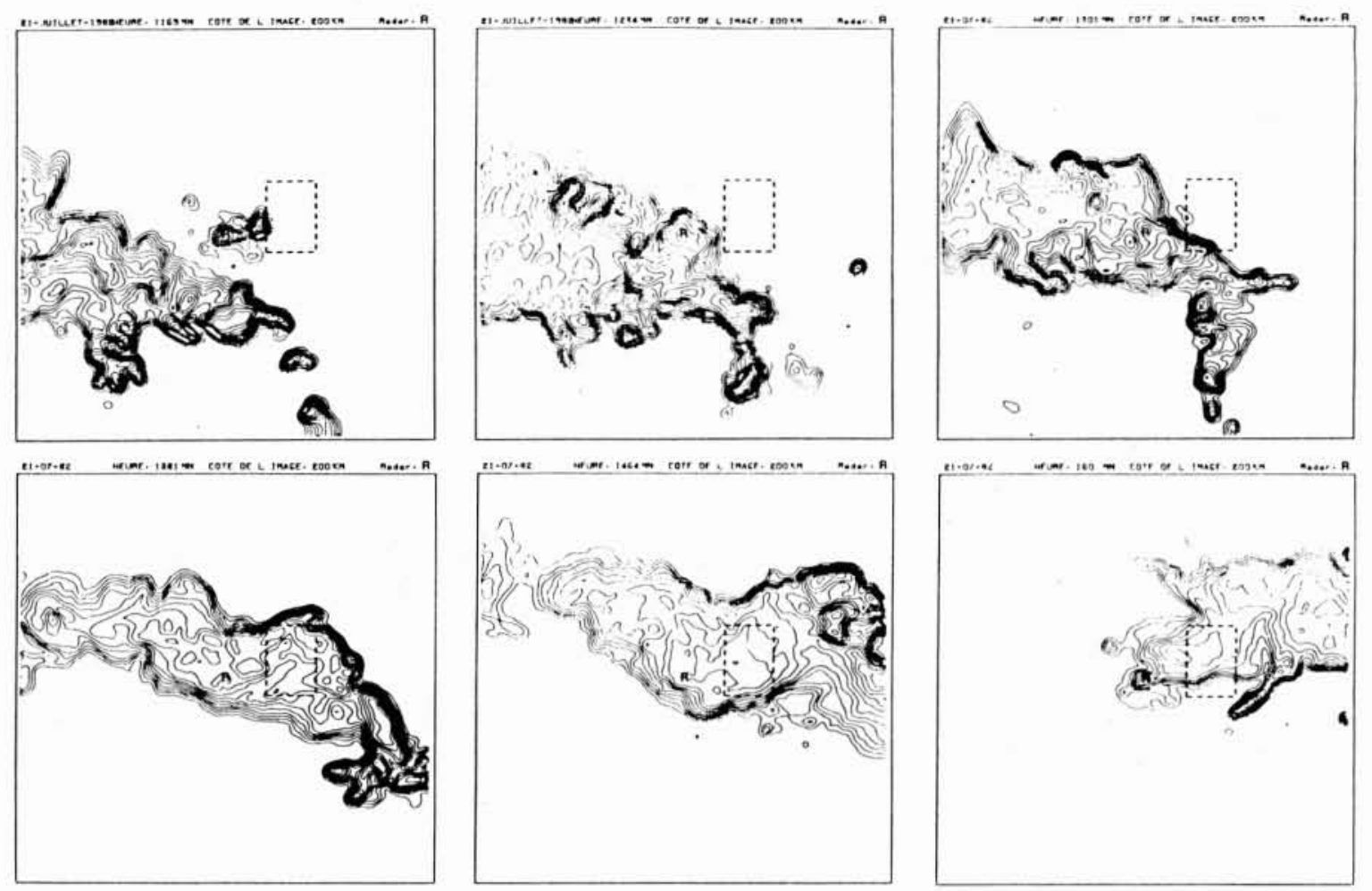

8.1. Déroulement de la séquence pluvieuse du 21 juillet (les images sont séparées par un intervalle de temps voisin d'une heure). Le rectangle pointillé schématise la zone d'étude.
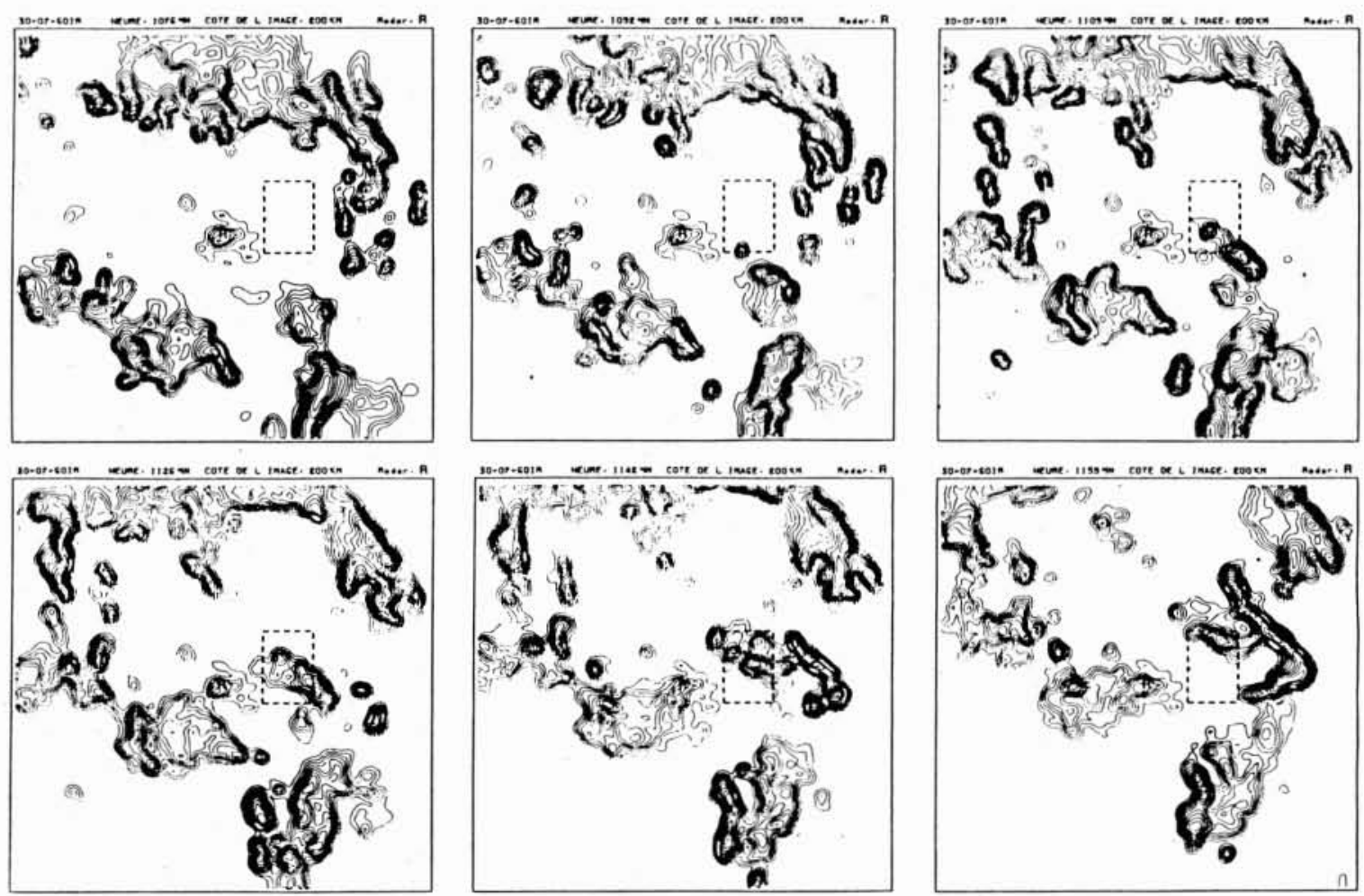

8.2. Déroulement de la séquence pluvieuse du 30 juillet au soir (les images sont espacées de 15 minutes environ). 


\subsection{Le radar, instrument de prévention et de prévision}

Le fonctionnement courant d'un Service Météorologique fait appel à une information météorologique que le radar peut pour partie satisfaire. La couverture radar améliore la sécurité des personnels qui interviennent dans le réseau. Une annonce préventive du risque de pluie est prépondérante pour déclencher les alertes à bon escient. Il devient alors possible de mieux préparer le réseau à l'importance de l'événement attendu, en vidant par exemple les bassins de rétention. Dans le cadre du projet de gestion automatisée, il est bénéfique de disposer d'informations plus quantitatives sur l'évolution des précipitations : heures de début et de fin de pluie, intensité moyenne, et si possible, prévision des intensités pour la demi-heure ou l'heure qui suit (Frérot, 1984). Des travaux sont en cours sur ce sujet (Einfalt et al., 1984).

La figure 8 , qui retrace le déroulement de deux des séquences pluvieuses de l'été 1982 apporte des éléments sur le rôle que pourrait jouer le radar. Le 21 juillet, la zone pluvieuse est détectée plusieurs heures avant le début de la pluie et un déplacement régulier est noté; l'importance de la zone pluvieuse peut également être appréhendée. Dans cet exemple, il parait possible dapprocher avec anticipation l'heure de début de la pluie et un ordre de grandeur de la lame d'eau. Il en va différemment pour les orages du 30 juillet au soir. Le radar montre le développement d'un essaim de cellules pluvieuses qui enserrent la zone d'étude et l'atteignent; toute prévision paraît exclue. Les images mettent cependant en évidence le risque d'èclatement des orages et autorisent des mesures préventives.

Depuis 1984, le Service d'Assainissement dispose d'un déport de l'image radar de Dammartin (Seine-et-Marne) qui confirme cet intérêt qualitatif (Jacquet, 1984). En prenant également en compte les résultats encourageants obtenus, il a été décidé d'intégrer l'image radar au projet de gestion automatisée.

\subsection{Le radar, instrument de mesure des intensités en temps réel}

Cette décision est importante car elle garantit la poursuite, dans des conditions favorables, des études et recherches relatives à l'intérêt hydrologique du radar. En effet, l'étude d'un nombre limité de séquences pluvieuses est insuffisant pour cerner les atouts du radar, les difficultés qu'il soulève et pour définir des conditions d'exploitation opérationnelles. Dans cette perspective opérationnelle, il est prépondérant de bien maitriser les anomalies susceptibles d'affecter l'image radar, qui peuvent apparaître lors des orages locaux. La constitution progressive d'une banque de données incluant images radar, mesures pluviographiques et limnigraphiques fournira les éléments d'analyse. L'objectif poursuivi est en effet d'utiliser, en temps réel, les images radar et les pluviographes télé-transmis pour définir le champ pluvieux affectant la Seine-Saint-Denis. Cet objectif ne sera atteint que très progressivement, en affinant des modalités d'exploitation des images, modalités qui seront testées en temps différé sur une série de séquences pluvieuses. Cette prudence paraît nécessaire dans un domaine nouveau pour les hydrologues. Elle ne doit pas masquer l'intérêt potentiel du radar qui, calibré de façon très simple, nous autorise une qualité d'estimation voisine de celle qui nous est accessible actuellement. Les résultats obtenus lors des pluies de l'été 1982 donnent quelques indications sur les conditions d'exploitation du radar en Seine-Saint-Denis :

- fréquence d'acquisition au moins égale à une image toutes les cinq minutes,

- utilisation complémentaire du radar et de la totalité des pluviographes télétransmis (dans une première phase), - calibrage de l'image radar au moyen d'un facteur moyen de correction réévalué à chaque pas de temps. Le risque d'erreur paraît plus élevé lorsque moins de trois pluviographes participent à ce calibrage.

\section{Radar météorologique et simulation des débits}

\subsection{Présentation}

Toute l'approche précédente a été consacrée à l'estimation des précipitations à l'aide du radar, en adoptant comme référence la mesure pluviographique. L'intérêt hydrologique du radar doit être apprécié en liaison directe avec le bassin versant, par l'aide qu'il peut apporter pour évaluer les débits engendrés par une pluie donnée. En effet, une mesure parfaite des intensités n'est pas nécessaire pour représenter correctement l'évolution des débits. Sous certaines hypothèses, le modèle de ruissellement lisse et réduit les incertitudes initiales sur les précipitations; une approche analytique très simple permet de le vérifier.

En hydrologie urbaine, la présence d'un enregistreur pluviographique sur un petit bassin versant constitue une situation très favorable. Par rapport à cette référence, l'utilisation du radar introduit une erreur dans la mesure des intensités. Cette erreur relative peut être caractérisée à partir des résultats précédents; ses réalisations successives en un point sont décorrélées, elle est sans biais, normalement distribuée et donc définie par sa variance $\sigma p^{2}$, dont la valeur dépend de l'origine météorologique des précipitations. Si l'on considère que le bassin versant peut être représenté par un modèle linéaire réservoir simple, largement utilisé en hydrologie urbaine, il est possible de relier l'erreur initiale à l'erreur résultante sur les débits; cette dernière se caractérise également par sa variance $\sigma q^{2}$. L'aptitude du modèle à filtrer les erreurs d'intensité peut être décrite par un paramètre de gain d'écart: $G=$ $\sigma p / \sigma q$; le rôle du modèle sera d'autant plus sensible que $G$ sera supérieur à 1 . Ce paramètre évolue en permanence au cours d'une séquence pluvieuse; pour un pas de temps $\Delta t$ donné, sa valeur croît avec le temps de réponse du bassin versant.

A titre d'exemple, le gain d'écart $G$ a été déterminé pour le débit maximal provoqué par un hyétogramme triangulaire symétrique. Pour un pas de temps de $10 \mathrm{mn}$, la figure 9 (page suiv.) illustre les variations de $G$ en fonction du temps de réponse $(\mathrm{K})$ du bassin versant et de la durée de la pluie.

Cette approche analytique simple précise le rôle de filtre du bassin versant. Elle donne en outre un ordre de grandeur de la précision espérée sur les débits simulés en fonction de l'origine des précipitations et des caractéristiques du bassin versant. 


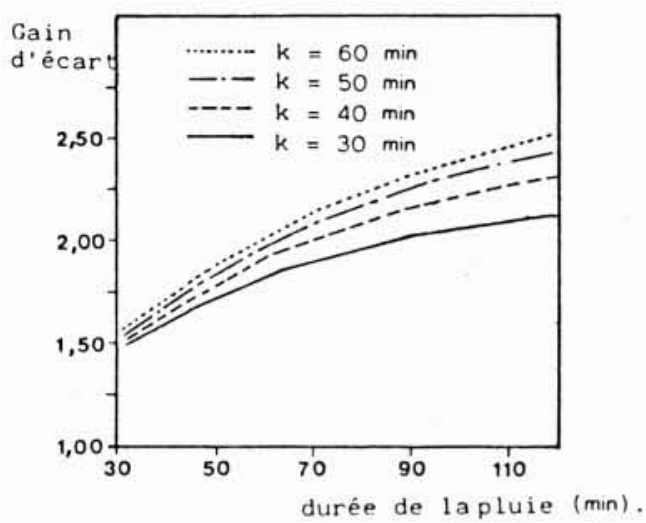

9. Evolution du gain d'écart.

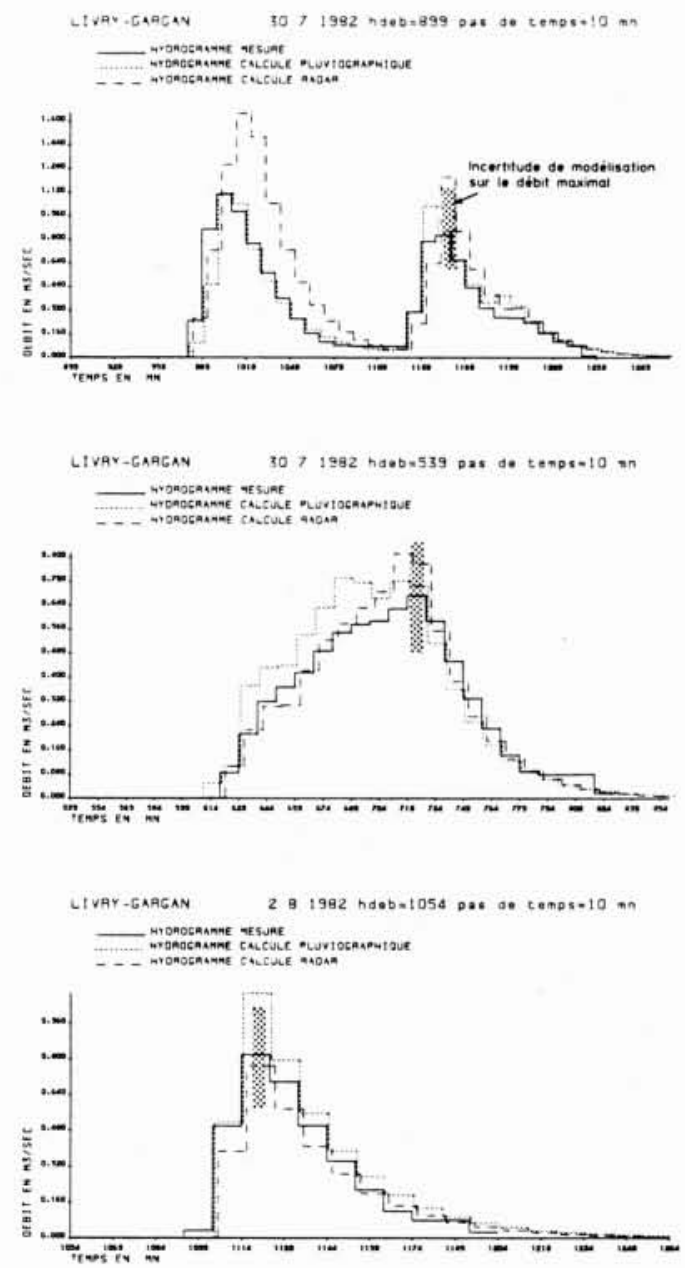

10. Comparaison d'hydrogrammes déduits de mesures radar ajusté et pluviographiques.

\subsection{Exemples de simulation de débits à partir d'images radar}

Trois bassins versants du département de Seine-SaintDenis sur lesquels un modèle de ruissellement avait été préalablement calé ont été retenus. Pour chacun d'entre eux, les débits consécutifs aux pluies de l'été 1982 ont été simulés en adoptant deux hypothèses :

- les intensités sont données par le pluviographe présent sur le bassin versant, situation très favorable,

- les intensités de la pluie sont déduites des images radar calibrées sans prendre en compte le pluviographe précédent.

Par rapport à l'approche précédente, sont introduites les incertitudes de modélisation. Les résultats obtenus pour le bassin versant de Livry-Gargan sont illustrés par la figure 10. Lors de la pluie régulière du 30 juillet au matin, les deux hypothèses conduisent à une reproduction correcte de l'hydrogramme, qui traduit de plus la bonne adaptation du modèle. Dans le cas du 30 juillet au soir, la mesure pluviographique autorise une restitution plus fidèle du débit réel; mais lors de l'orage du 2 août, la mesure radar prend distinctement l'avantage.

Les deux hypothèses testées se révèlent donc équivalentes pour le bassin versant de Livry-Gargan. Dans les autres cas traités, il apparaît que les incertitudes de modélisation sont prépondérantes devant les écarts consécutifs aux hypothèses testées. En se basant sur les exemples traités en Seine-Saint-Denis, les intensités déduites des images radar sont susceptibles d'une utilisation comme entrée d'un modèle de ruissellement.

\section{Conclusion}

Cette étude s'est déroulée dans le cadre du projet de gestion automatisée du réseau d'assainissement de SeineSaint-Denis et avait pour but de préciser l'intérêt du radar météorologique. Elle a permis de mieux cerner les atouts de cet instrument, les problèmes qu'il soulève, et doit être perçue comme une première étape dans la valorisation des images radar en hydrologie urbaine.

Un examen qualitatif des deux types de mesures, dont la cohérence a été étudiée, révèle quelques anomalies sur les images radar notamment lors des orages locaux; il apparaît également que les caractéristiques des images actuellement diffusées sont mal adaptées à l'hydrologie.

La qualité de l'estimation radar des intensités pluvieuses a été appréciée au niveau de la maille, donnée radar élémentaire. Les premières conclusions ne sont valables que pour la Seine-Saint-Denis et le réseau de mesure existant. Cette qualité d'estimation dépend directement de la variabilité des champs pluvieux; les orages 
locaux étant plus difficiles à restituer que les pluies homogènes. La continuité spatiale introduite par les images radar n'est donc pas totalement valorisée. La comparaison de plusieurs méthodes de calibrage montre leur relative équivalence, pour les exemples étudiés. Il s'avère également qu'interpolation pluviographique par krigeage et calibrage des images radar conduisent à des résultats voisins. Cette constatation confirme que nous ne sommes pas en mesure d'exploiter au mieux l'image radar; elle montre également que, calibré de façon simple par un facteur moyen de correction, le radar procure une cartographie des intensités proche de celle accessible par des techniques d'interpolation évoluées dont l'application opérationnelle n'est pas immmédiate. Les travaux devront se poursuivre sur ce sujet; ces premiers résultats sont cependant encourageants en faveur du radar.

La place du radar comme instrument de prévention et de mesure dans le projet de gestion automatisée de Seine-Saint-Denis a été abordée. L'intérêt hydrologique du radar doit également être appréhendé en liaison directe avec le bassin versant par l'aide qu'il peut apporter pour simuler des hydrogrammes de crue. Une approche analy- tique simple a permis de préciser le rôle de filtre que le modèle de ruissellement joue vis-à-vis des erreurs sur les intensités pluvieuses. Des exemples concrets sur des bassins versants de superficies variées montrent que l'estimation radar ou la mesure pluviographique sur le site conduisent à une qualité voisine de simulation des débits.

\section{Remerciements}

Cette étude est le fruit d'une collaboration entre le L.C.P.C. et le CERGRENE, chargé de l'élaboration du logiciel d'aide à la conduite du projet de gestion automatisée du réseau d'assainissement de Seine-Saint-Denis. Nous tenons particulièrement à remercier $M$. André Bachoc, responsable de la Subdivision de gestion des eaux à la DDE de Seine-Saint-Denis, M. Patrick David, Ingénieur de la Météorologie nationale, qui a assuré l'acquisition des images radar, et M. Renaud Sanejouand, Directeur technique du L.C.P.C. qui a grandement facilité cette collaboration.

\section{Références bibliographiques}

ANDRIEU H. - Interprétation des mesures du radar Rodin de Trappes pour la connaissance en temps réel des précipitations en Seine-Saint-Denis, Thèse de Docteur-Ingénieur, ENPC (1986).

Austiv GL. et AUstin LB. - The use of radar in urban hydrology. Journal of Hydrology. 22. 1974. pp. 131-142.

BACHOC A. - Disons douze ans ! Pour commencer à automatiser la gestion du réseau d'assainissement de Seine-Saint-Denis. AO $\mathrm{n}^{\circ} 3,1984$.

BRANDES EA. - Optimizing rainfall estimate with the aid of radar. Journal of Applied Meteorology; Vol. 14, oct. 1975 , pp. $1339-1345$

Collier CG., Cole JA. and Robertson RB. - The north West Weather Radar Project : the establishment of a weather radar system for hydrological forecasting. Actes du Coloque d'Oxford. Avril 1980. IAHS Publ. n' 129, pp. 31-40.

COLLIER CG. - Accuracy of Rainfall Estimates by Radar. Journal of Hydrology, 83 (1986), p. 207-249.

DAvid P. - Principe de fonctionnement du radar météorologique Météorologie Nationale.

Dee Weather Radar and real time hydrological forecasting project. Central Water Planning Unit. nov. 1977.

Delnomme JP. - Application de la théorie des variables régionalisées dans les sciences de l'eau. Thèse de Docteur-Ingénieur. Ecole des Mines de Paris, 1976.

DELRIEU G. - Evaluation d'un radar météorologique pour la mesure des précipitations. Validation et étalonnage technique géostatistique; application au Bassin Parisien. Thèse de Docteur-Ingénieur, INP Grenoble, 1986
DUPOUYET JP. - Le radar et la pluie. Application à l'annonce et à la prévision des crues dans les bassins critiques de la Vézère et de la Corrèze, La Houille Blanche, 1983, n' 5/6, pp. 387-392.

Einfalt T., Schilling W. - SSCOUT - A Storm Tracking Procedure for a Micro Computer. 3 International Conference in Urban Storm Drainage. Göteborg 1984.

FrÉrot A. - Procédures d'optimisation des consignes de gestion d'un réseau d'assainissement automatisé. Thèse de doctorat, ENPC (1987)

Froment G. - Rapport Hydromel. Météorologie Nationale. 1981.

Gilet M. et CiCcione M. - Le projet Aramis et la prévision à courte échéance. La Houille Blanche, 1983, n'5/6, pp. 367-376.

Harrold TW., ENGlish EJ., Nicholass CA. - The accuracy of radar derived rainfall measurements in highly terrain Quart. J.R. Met. Society (1974), 100, pp. 331-350.

HUFF FA. and TOWERY NG. - Utilization of radar in operation of urban hydrologic systems. 18th Radar Meteorology Conference. AMS Atlanta, pp. 437-441.

JACQUET G. - Mise en place d'un système d'alerte météorologique. Rapport CERGRENE, déc. 1984.

MATHERON G. - La théorie des variables régionalisées et ses applications. Fascicule 5. Ecole des Mines de Paris. 1970.

Sauvageot H. - Radar Météorologie, Ed. Eyrolles, 1918.

Sauvageot H. - Mesures hydrologiques par radar. La Houille Blanche. $1983, \mathrm{n}^{\circ} 5 / 6$, pp. $329-340$.

WILSON JW., BRANDES EA. - Radar Rainfall measurement : a summary. Bulletin of American Meteorological Society. Vol. 60 , sept. 1979, pp. 1048-1058. 